\title{
A conceptual framework for designing smart learning environments
}

\author{
Sirkka Freigang ${ }^{1 *}$, Lars Schlenker ${ }^{2}$ and Thomas Köhler ${ }^{2}$
}

\author{
* Correspondence: \\ sirkka.freigang@bosch-si.com \\ ${ }^{1}$ Bosch Software Innovations, Berlin, \\ Germany \\ Full list of author information is \\ available at the end of the article
}

\begin{abstract}
The paper deals with the design of Smart Learning Environments (SLEs). Over and above that, it's about the interconnection between SLEs and the internet of things. With the question of how an interdisciplinary approach can contribute to the design of SLEs together with the disciplines of education, computer science and architecture the study empirically applies data collected in 2016. The empirical research was initiated by consolidating the findings from transdisciplinary analyses of the literature in a hypothetical model for designing SLEs. This model pooled success factors from different spheres of influence and served as a tool for empirical research. A triangulation was used for facilitating a model-validation in the context of 7 expert interviews, 7 questionnaires as well as a workshop with training experts. The data analysis resulted in the development of a holistic, socio-technical framework.
\end{abstract}

Keywords: Smart learning environments, Further education, Interdisciplinarity, Internet of things, Development framework, Workplace learning

\section{Introduction}

Lifelong learning will change as smart technologies increasingly support the acquisition of skills in the workplace (Ebner et al. 2011). This will call for connected, state-of-the-art learning environments that inspire the imagination, spark creativity and motivate learners to tackle content on their own. These challenges open up new opportunities for educational innovations such as smart learning environments, which could be seamlessly integrated in our workplace. This paper seeks to offer new science-based answers to the knowledge society's challenges. Tomorrow's knowledge is sure to be connected, distributed and interdisciplinary. A holistic, big-picture view of education as a whole best reveals the opportunities and possibilities inherent in digital technologies, particularly as personalized learning processes emerge. The digitalization of education is pervasive; it permeates all educational processes, places and formats (Kerres 2016). This challenge is about people's needs rather than technological possibilities.

Rapid advances in technologies such as the Internet of Things, big data and data sciences, robotics, human-machine interaction, the user experience, artificial intelligence, embedded systems, and the like provide the technical underpinning for designing complex, technology-enhanced learning solutions such as adaptive and ubiquitous learning scenarios (Brusilovsky and Peylo 2003; Erpenbeck and Sauter 2013, 2015; Winkler et al. 2014).

(c) The Author(s). 2018 Open Access This article is distributed under the terms of the Creative Commons Attribution 4.0 International License (http://creativecommons.org/licenses/by/4.0/), which permits unrestricted use, distribution, and reproduction in any medium, provided you give appropriate credit to the original author(s) and the source, provide a link to the Creative Commons license, and indicate if changes were made. 
This paper presents the latest findings of an empirical study conducted to illuminate the role of smart learning environments (SLE) and their design requirements while factoring the aforementioned framework conditions for didactically-based adult education models into the equation.

\section{Study design}

This research project aimed to find out if and how the Internet of Things is applicable to teaching and learning processes in smart learning environments in a way that sustainably supports learning at the workplace. This paper thus focuses on corporate learning spaces that offer the best opportunities for lifelong learning and seamlessly combine informal and formal learning. Its authors chose an interdisciplinary approach that centered on the interlaced disciplines of education, computer science and architecture/design to answer 2 main research questions (with 6 sub-questions):Research question 1: What new teaching and learning formats are created by the Internet of Things?Research question 2: How can the physical environment support the learning processes? Furthermore, the research aims to develop a didactically sound concept for designing intelligent and hybrid learning spaces like SLEs.

This investigation entails a series of studies that build on and complement one another in a multi-method study design geared to compensate for the drawbacks often associated with mixed methods. The study design with this multi-stage triangulation provides a more comprehensive picture than a single study could have done. The authors subdivided the study design into two subordinate studies and one main study and combined qualitative and quantitative methods of social research.

In the first step, literature reviews substantiated the connection between the theoretical constructs of the Internet of Things and smart learning environments. To this end, the authors systematically deconstructed known and directly related learning methods to transparently demonstrate that IoT-based learning forms such as smart learning environments (SLEs) are a specific combination of adaptive and ubiquitous learning formats that interact so as to facilitate new functions and possibilities at the workplace.

In a second step, the authors initiated the empirical investigation by condensing the insights gained from reviews of transdisciplinary literature into a first hypothetical model for designing intelligent and hybrid learning spaces. This model consolidated success factors sourced from various spheres of influence and served as a search grid for the empirical study. The modeling process based on a common method for modeling information systems according to Hevner et al. (2004). This framework (Hevner et al. 2004) forms a clearly recognizable socio-technical perspective in comparison to other modeling methods (Stachowiak 1973) and can thus best meet the situation of educational information systems. In this way the authors combined and condensed philosophical, psychological and technological approaches in a deliberate way along the lines of Spector's "preliminary framework for smart learning environments" (Spector 2014). In a third step this first model was validated through seven expert interviews (main study) which were combined with 7 questionnaires (sub study 2). The problem-based, guideline-supported expert interviews were conducted with renowned scientists from the field of computer science, architecture/design and education in Germany. The main study addressed the following in addition to the already mentioned research questions: 
Sub question 1.1: How can learning processes be supported by the Internet of Things?

Sub question 2.2: What are the design requirements for learning spaces?

The interviews were conducted personally (face2face) and usually took over $1 \mathrm{~h}$. The aim was to obtain a comprehensive expert opinion on the model and to classify the identified areas of influence with the respective success factors for their importance in the design process. The interviews were recorded, transcribed and coded with MAXQDA. For the data analysis, the content-structuring content analysis according to Mayring (2010) was used. Sub-study 2 was a fully standardized quantitative survey tool that complemented the qualitative interview on the basis of a presentation and evaluation of six areas of influence and a total of 47 items (success factors). The data collection based on the following research questions:

Sub question 1.2: Which areas of influence characterize intelligent and hybrid learning spaces?

Sub question 1.3: Which success factors have to be considered when designing intelligent and hybrid learning spaces?

Sub question 1.4: What could a didactically sound model for the design of intelligent and hybrid learning spaces look like?

The data analysis of sub-study 2 was conducted with SPSS. The qualitative survey was combined with a quantitative questionnaire to systematize the expert opinions, to be able to compare them and to enable a quantifiable evaluation. The qualitative and quantitative data collection took place simultaneously during the interview situation. To complete the validation phase, sub-study 1 was carried out within a workshop. The target group was defined as corporate learning professionals, people with a lot of experience in continuing vocational training. The workshop was attended by 6 trainers and a head of training department. The aim of sub-study 1 was to receive suggestions and feedback from practitioners in addition to the academic perspectives.

In the last step, this first hypothetical model was redesigned and modified on the basis of merging all empirical data into one holistic, socio-technical framework.

The preceding section discussed this research effort's grounding in educational science (cf. section "Study design"); the next section will break down the object of investigation (SLEs) along the lines of neighboring disciplines.

\section{Current state of research into smart learning environments}

Research into SLEs is an interdisciplinary pursuit. Smart learning environments are the object of research in the field of (business) computer science as well as in other socio-scientific disciplines such as (media) education and psychology. Approaches to and definitions of SLEs vary accordingly and will be discussed below. To review the literature, the authors selected papers that deal explicitly with the concept, development, success factors and requirements for SLE design processes. They did not consider papers on the broader subject of smart learning, as it is beyond the scope of this study. A systematic review of literature up to September 2016 identified a total of five key papers to be examined, compared and discussed below. 
An article by Lei et al. (2013) merits mention as a precursor to the SLE research published in the Journal for Smart Learning Environments since 2014. That essay is relevant to this study because its authors develop smart learning environments based on cyber-physical systems, which would indicate a connection to IoT rarely mentioned in literature. Lei et al. (2013) described an SLE at a university that is enabled by "smart applications." To this end, a cyber-physical system used embedded components to connect various types of sensors and actuators and automatically control specific functions. It controlled and automatically adapted external conditions that are conducive to learning, such as lighting, heating and air quality, and significantly increased energy efficiency (Lei et al. 2013). This paper illustrates the relative ease with which "smart functionalities" may be integrated into learning spaces and conditions conducive to learning can be supported.

As part of a "preliminary framework for smart learning environments," Spector (2014) combined philosophical, psychological and technological approaches to develop a platform for planning and implementing SLEs. In this author's view, smart learning environments have to be significant and have to achieve sustainable educational work at the levels Engagement, Effectiveness and Efficiency (3Es). Spector (2014) advocates more strategic planning and design of SLEs and points out that one must first consider how human learning works and how people actually acquire knowledge and skills. This author points to insights into educational psychology that need to be systematically integrated into the design process. According to Spector (2014), technology has to support the "3Es" listed above to lend this technology meaning and a tangible benefit. Putting the significance of SLEs into the greater context of social responsibility, Spector (2014) suggests that SLEs should help people become thoughtful, productive and considerate members of society.

Spector generically describes a meta-concept similar to a deductive approach, with a perspective from the outside looking in. Koper (2014), in contrast, developed a new theoretical concept for the design of SLEs rooted in concrete interactions between humans and technology. Koper (2014) calls this concept, which is based on a more inductive approach and ties into the design of user interfaces (human-computer interaction), "human learning interfaces (HLIs)." To apply lessons learned from user interfaces to human learning interfaces within SLEs, Koper (2014) first distinguishes between different learning environments by the degree of digital and analog learning stimulus.

Concluding that SLEs are to be classed as "embedded cases," Koper (2014) outlined prototypical requirements for this specific learning environment to provide the basis for developing SLEs:

1. Digital devices are added to physical learning locations

2. Digital devices detect the learner's location and context

3. Digital devices augment the physical learning environment with additional digital learning functions

4. Digital devices monitor the learner's progress

According to Koper, smart learning environments aim to enable faster and better learning. He analyzed the conditions that would facilitate this and developed a concept for "human learning interfaces" (HLIs) based on the "modal model of the architecture 
of human information processing" (Ashcraft and Radvansky 2010). Koper (2014) distinguished between five different HLI types each with specific learning objectives (revising \& restructuring, improving \& rendering, generating new cognitive representations) and learning forms (reflection, practice, identification, socialization, creation). Based on these references, Koper extended his definition of smart learning environments as follows (Koper 2014 p. 14):

"SLEs are physical environments that are improved to promote better and faster learning by enriching the environment with context-aware and adaptive digital devices that, together with the existing constituents of the physical environment, provide the situations, events, interventions and observations needed to stimulate a person to learn to know and deal with situations (identification), to socialize with the group, to create artefacts, and to practice and reflect."

The HLI concept presented by Koper (2014) is particularly well-suited for developing specific SLE use cases and the associated IT system architecture on mobile devices, which could be implemented via an app on a smartphone, for example. Unfortunately, elementary and detailed forms of learning, especially in interaction with other teachers and learners, are hardly illuminated and merely outlined. Questions about the design of the physical learning space that is to stimulate learning processes and about the organizational conditions and factors influencing SLEs' introduction also remain unanswered.

In his framework for SLEs, Hwang (2014) first exhaustively covered the terminology used and pointed out that combined adaptive and ubiquitous forms of learning enable "smart learning." Hwang (2014) transparently deconstructs and compares the terms "smart learning," "ubiquitous learning" and "adaptive learning" in a table.

In ubiquitous learning, an information system is able to identify the learner's situational context and place to provide learning functions tailored to the circumstances. If this is interlaced with adaptive learning, this means that the system also knows the learner's needs and can exploit these data to offer various informational and learning methods, that cater to the given type of learner like "supportive tools or learning suggestions in the right place, at the right time and in the right form" (Hwang 2014 p. 2). For Hwang (2014) an SLE needs to be context aware, adaptive and personalized to best fit learners' needs. Building on this foundation, Hwang (2014, p. 5) formulated the following definition for SLEs:

"Smart Learning Environments can be regarded as the technology-supported learning environments that make adaptations and provide appropriate support (e.g., guidance, feedback, hints or tools) in the right places and at the right time based on individual learners' needs, which might be determined via analyzing their learning behaviors, performance and the online and real-world contexts in which they are situated."

Compared to the SLE approaches described above, Hwang's SLE framework is conspicuously technical, conveying the impression that it caters mainly to system 
developers. In closing, Hwang set out various research requirements relating mainly to educational matters. He also pointed out that SLEs can only be developed and implemented in collaboration with experts in various disciplines, particularly computer science and education. This section wraps up the discussion of presented SLE concepts with a look at a paper by Begoña Gros (2016) in which she expounds on the potential for self-directed learning in SLEs and advocates a participatory design process for SLEs.

According to Gros (2016), the widespread use of mobile devices such as smartphones enables ubiquitous learning. She also pointed out that use of the mobile device is self-determined. While a teacher generally controls formal learning scenarios, this is not the case when learning with mobile devices. Gros stated that SLEs aim to orchestrate between formal and informal forms of learning. In her opinion, this requires new educational approaches that view learning as a subjective, situated, social and very dynamic construct that manifests itself differently in each learner.

Building on the ten properties of SLEs designated by Zhu et al. (2016) (1. Location-Aware, 2. Context-Aware, 3. Socially Aware, 4. Interoperability, 5. Seamless Connection, 6. Adaptability, 7. Ubiquitous, 8. Whole Record 9. Natural Interaction and 10. High Engagement) Gros concluded that SLEs' main purpose is to offer support suitable for and tailored to the learner. According to Gros, an SLE takes on the role of a coach or a learning guide and is based on the following educational principles:

1. Conversation

2. Reflection

3. Innovation

4. Self-organization

The learning environment can automatically organize content and methods and, over a prolonged period, improve learning performance. Building on Barron's learning ecologies framework, Gros stated that learners are the main protagonists of smart learning and that they are responsible for social relationships and creating significant constellations in the physical and virtual learning context. Gros thus advocates a human-centered approach to designing SLEs that specifically addresses learners' needs. She noted that viable SLEs can only be developed with the knowledge of what "smart learners are" and what motivates them.

In contrast to the other authors, Gros did not present an SLE design concept of her own, instead shedding light on the design process by referencing current SLE research. She concluded that the application of participatory design methods contributes to the development of SLEs that can cater to learners' needs and socio-cultural backgrounds.

\section{Limits of earlier SLE research}

To sum up the state of research, SLEs are predominantly uniform in function and features. They are able to identify their environment and provide learners with situational and demand-driven content, learning strategies and tools. There is also a broad consensus on the need to research and develop SLEs across disciplines, in particular by combining educational and IT knowledge. However, the individual authors' diverging approaches merit mention. Their concepts address different levels; their conceptual 
approaches and theoretical references vary. Nevertheless, the presented approaches, concepts and frameworks for designing SLEs furnish relevant insights on which this paper builds. It can also be said that the literature on SLEs does not address architectural design principles despite the fact that physical learning spaces are a recurring topic. And there are deficits in organizational aspects and framework conditions that impact SLEs' implementation. As a general observation, there is notable absence of a holistic, interdisciplinary concept that adopts a socio-technical perspective and provides a systematic, science-based procedure for designing SLEs.

\section{Theoretical relationships between SLEs, internet of things (IoT) and work- place learning}

This paper's authors soundly deconstructed, categorized and compared different definitions of the term "Internet of Things" to gain science-based insight. They analyzed the literature in depth to distill the meanings and assess definitions and descriptions of the term "Internet of Things" (IoT) from 26 sources. The term stems from the Auto-ID Center at the Massachusetts Institute of Technology (MIT), which designed the inceptive intercompany RFID infrastructure in 1999. Kevin Ashton, co-founder and then head of the Auto-ID Center, was the first to use the phrase "Internet of Things" Fraunhofer (2018). Ashton envisioned that computers should be able to gather information independently of humans. Computers would have to understand the real world without human operators. This would then prevent many losses and expenses.

The notion of an Internet of Things is rooted in the concept of "ubiquitous computing" (Weiser 1991) outlined by Mark Weiser in the early 1990s. Mark Weiser formulated first visions of an ambient intelligence, integrated in the (future) workplace, in which all things and tools are connected with each other and are able to communicate or send emails for example. Other researchers picked up on the idea and developed it further in the ensuing years. The idea behind ubiquitous computing is that microelectronic components can serve to extend any physical object beyond its existing form and function; the same goes for the very similar terms "pervasive computing" (Estrin et al. 2002; Lucke and Rensing 2014) and "ambient intelligence" (Aarts and Harwig 2002). This creates "smart" objects equipped with digitally linked process logic, sensors and connectivity, which in turn create an Internet of Things in which the computer vanishes as it is assimilated into the "smart objects" of the physical world (Mattern and Fleisch 2005).

As their name would suggest, these "smart objects" are a key feature of the Internet of Things. They are created by enriching everyday objects with "technical intelligence." The authors Fleisch and Thiesse (2014) describe this special feature in this way:

"The Internet of Things describes the idea of an extended Internet, which, in addition to classic computers and mobile terminals, also integrates any physical object into its infrastructure by means of sensors and actuators, thereby making it a provider or consumer of the most diverse digital services."

The Internet of Things connects the physical world of objects with the virtual world of data (Vermesan and Friess 2015). Applied to educational purposes, this means that the Internet of Things connects the physical world of (learning) objects with the virtual 
world of personal learning environments and it's learning histories. It will culminate in a full-fledged merger, a hybridization of the analog and digital worlds. Boundaries blur and can no longer be clearly separated. Smart objects / things are able to perceive their environment and process information. This "technical intelligence" can be very diverse, ranging from rather more passive acquisition, storage and processing of information to autonomous actions in response to their environment and interaction with users in an embedded system. RFID, sensors and actuators provide the technological prerequisites for this. In summary, smart objects can be said to be ubiquitous, invisible and autonomous (Gabriel et al. 2010).

A review of educational literature using secondary IoT search terms revealed that smart learning environments are equated with IoT-based forms of learning. It can be concluded that the technological construct of the Internet of Things, with its origins in ubiquitous computing and ambient intelligence, can be combined with the learning construct of SLEs, as SLEs are also rooted in the concepts of ubiquitous learning, pervasive learning and adaptive learning. Hence, the Internet of Things facilitates specific characteristics of SLEs such as context sensitivity.

These analyses also show that the Internet of Things is applicable to teaching and learning processes. This creates new teaching and learning formats that scientists call "smart learning environments" (SLEs). This study is the first research effort devoted explicitly to applying the Internet of Things to on-the-job teaching and learning processes in SLEs with a focus on workplace learning in Germany.

Digitization paves the way for new technologies, such as the IoT. On the other hand, digitization leads to a higher need for continuing education in companies. Lifelong learning is becoming more important. Parallel to this, traditional and highly formalized continuing education seminars are increasingly branded as too expensive, not very effective and too little practice-relevant. Therefore, new approaches like IoT-based learning scenarios are required, which are increasingly pushing self-directed learning on demand in the workplace and appear as informal learning formats. SLEs are defined to support learning at the right place, at the right time and on the right form (Hwang 2014). In addition, SLEs are focusing on self-directed and situated forms of learning. Therefore, SLEs seem to be able to pick up these current needs in a new educational solution, in order to merge the existing gaps between formal and informal learning and to support life-long learning at the workplace.

\section{Theoretical conclusions on SLEs, loT and physical learning nodes}

This section provides a summary of key spatial design principles in a bid to paint a comprehensive picture of the requirements for physical SLEs. These principles were sourced from reviews of literature in the fields of spatial sociology, spatial theory and learning space research (Eigenbrod \& Stang 2014; Knoll 1995; Sesink 2007).

Given the diversity of learning methods, the first priority for learning spaces is that they need to be versatile. They ought to promote creative teamwork and offer designated quiet areas where people can concentrate. They should also furnish plenty of space for interaction, dialog and presentations. Conventional classrooms are increasingly becoming "experimental workshops and research laboratories" (maker spaces) for design and innovation-based working methods. Employees need an environment conducive to developing and implementing ideas so they can challenge themselves and 
engage in experience-oriented learning. A relaxed atmosphere is important for creative phases. Doorley and Witthoft (2012) and d.school Stanford wrote a handbook for designing creative spaces that recommends specific pieces of furniture, materials and tools for collaborative work. The importance of lighting is glaringly obvious in modern working environments. Natural materials such as stone and wood combined with plants are very popular means of conjuring a pleasant atmosphere. Designers are also crafting living room-style office interiors to ensure people enjoy spending time there.

It can be said that interior design has an immediate impact on well-being and, by extension, on learning processes. Adaptable interior designs that enable variable use of rooms support the give-and-take between various phases of learning. Some areas of these rooms are designated for working alone; others for collaborative teamwork. The different room arrangements outlined above support the variety of learning methods, but specific arrangements or interior designs do not necessarily need to be realized in separate rooms.

Closely linked, overlapping digital and physical areas can transform a room that is conducive to learning as described above into hybrid learning spaces or even into a smart learning environment. The result of this mix is a new area that has new emergent properties of its own and is therefore more than the sum of its individual parts (Kohls and Münster 2017). Traditional learning spaces will be augmented with integrated, invisible technology (see section "Theoretical conclusions on SLEs, IoT and physical learning nodes") to expand these rooms into intelligent and hybrid learning spaces (SLEs). As pointed out in the previous paragraph, any equipment and learning objects can be enhanced with IoT components. This creates smart objects that are connected to one another and to the Internet so that they interact in a complex cyber-physical system.

An important criterion for connected and intelligent learning spaces is "unobtrusive technology." (Sesink 2007, p.3). This is where the potential of IoT technologies unfolds, for these miniaturized objects can generally be integrated inconspicuously into architecture and room equipment like furniture. In summary, it can be said that the Internet of Things is making deeper and deeper inroads into everyday (office) life. Initial studies have also substantiated its positive effects on teaching and learning processes. For example, Gomez et al. (2013) worked with QR codes and enhanced physical learning objects with digital information that learners retrieve via QR code scanners. With this relatively simple method, the authors were able to connect the physical and virtual worlds and enrich analog learning content with multimedia (Gómez et al., 2013). Another study has shown that automated control of external factors such as lighting, heating and cooling has a positive effect on learning processes (Uzelac et al. 2015). However, considering the possibilities inherent in the IoT, the empirical research is clearly still in its infancy.

A recent study (Kanagarajan and Ramakrishnan 2017) identified and compared all ubiquitous and environmentally intelligent learning infrastructures known worldwide. The authors classified the identified examples by the categories architecture, approach, analysis, model, application and device, and found that these examples are not as intelligent as they should be. They designated a "smartness level" to distinguish between different stages of development.

\section{A holistic framework as a tool for human-centered SLE development}

The Internet of Things offers a wide range of options to support lifelong learning in the workplace. However, didactically sound application examples have yet to be identified. In 
order to systematically tap the potential of IoT in learning spaces, it will first be necessary to fully understand the needs of teachers and learners to then design and test didactically sound use cases and, in a final step, funnel these prototypes into the development pipeline. Authors Domingo and Forner (2010) also emphasize the importance of user-centric design in a paper that examines the development of hybrid learning environments in the context of IoT and eLearning. They stress how useful interdisciplinary teams are to developing such complex learning arrangements.

The following section builds on the theoretical underpinnings discussed above to present the findings of this investigation. It is based on a redesign of the first design model validated in the empirical study.

These findings provide a comprehensive picture of IoT-based educational innovations that have been researched to date in separate disciplines and addressing disparate concepts. With a people-centric concept for the design of SLEs providing the framework, the authors addressed the gaps in earlier SLE research. To this end, they methodically deconstructed the unilaterally developed concepts, took a holistic view of the disciplines and SLE design areas, and combined these in a transformative process. They systematically meshed architectural design principles and organizational aspects with SLEs' didactical and IT features.

The aim of the socio-technical framework is to translate scientific findings into practical application by fostering educational innovations that control the SLE development process holistically, on a scientifically sound basis.

This study's findings show that SLEs depend on several factors that can be categorized in the following spheres of influence:

[0] Participatory corporate culture.

[A] User centricity.

[B] Didactical variety.

[C] Hybrid learning space.

[D] Hybrid learning assistance

This framework pools 30 success factors that are assigned to the five aforementioned design patterns and collectively represent the ideal smart learning environment. For each design pattern, there are six success factors that are to be fleshed out for effective SLE product development. Each design pattern also has an integrated maturity scale to enable in-depth analysis of the current stage of development.

The development process is sequential. It kicks off by analyzing and reflecting on the corporate culture (design pattern 0 ) to provide the foundation for the entire design effort. The corporate culture can hardly be constructed directly-for the most part, this is done indirectly via the other four fields-so this first pattern serves only for analysis and reflection. The next step is to actively work on factors for user centricity (design pattern A) and then build on this work to develop didactical variety (design pattern B). Then it is time to design the spatial aspects (design pattern C). The final step is to address the technological factors relevant to intelligent learning systems (design pattern D).

The authors recommend using this framework as a dynamic design tool and focusing on the design areas that show great potential and are relatively easy to put into 
practice. The researchers defined seven minimum requirements needed to progress in stages towards the goal of creating SLEs. By following these steps, developers can implement hybridized forms of learning at a lower level of IoT maturity:

1. Needs assessment [A1]

2. Toolkit-supported learning [B10]

3. Hybridized learning [B11]

4. Digital \& analog learning tools [C13]

5. Physically integrated IoT [C18]

6. Identification of learning objects \& content [D19]

7. Process-based integrated IoT [D20]

The SLE framework serves as an iterative planning, analysis and development tool that combines socio-technical levels, dimensions and areas of influence relevant to the design, and simultaneously defines the respective success factors as the ideal type of design patterns, which in turn systematize, map and thus reduce the complexity of SLEs in interdependent, reciprocal interaction.

The following subsection presents the individual design patterns and their respective success factors in the context of their systemic interaction.

\section{Design pattern [0] - Corporate culture}

Empowerment is a success factor that fosters a participatory corporate culture. This should be taken to mean strategies and actions that increase autonomy and self-management, thereby enabling employees to perform their jobs over the long term. The corporate culture determines if and how employees are empowered to pursue their professional development in a self-organized way rather than being directed or controlled by others. It makes a difference if employees are sent to a training course or given the opportunity to do so. It also makes a difference if employees are allowed to experiment and make mistakes or if they are compelled to follow strict procedures. Hierarchical communication structures often impede an open and transparent exchange. However, dialog can be promoted, for example, via an internal network such as an ESN. Also, goals set by the learner are more likely to be attained than goals set by someone else. "Interdisciplinary perspectives" that are conducive to initiating innovative, interdisciplinary learning projects also help transcend legacy silo structures. It will take new forms of leadership, rather than mere management, to promote learning in networks and from others. Enduring empowerment requires an empathetic style of leadership based on trusted collaboration.

\section{Design pattern $[A]$ - Human centricity}

Smart learning environments are user-oriented (see section "Current state of research into smart learning environments"). And the design process must be geared to the learner to ensure SLEs are indeed user-centric. The point of departure for designing smart learning environments has to be the needs of the learners who use these spaces. This is why the human-centric design pattern (A) is the inceptive phase of the design process. Learning needs and learning offerings have to be a good match to foster 
"sound intrinsic motivation [A5]" and interest. "Activity and context analyses [A1]" should to be conducted in advance to learn which technical and methodological skills will be desired or necessary and to align these skills with corporate goals for the purpose ensuring future "employability [A2]." The bottom-up approach is for employees to set goals for themselves. The top-down approach is for the organization to set strategic goals. The two need to be compared and combined in a balanced way to create a (skills) profile. This "profiling [A3]" may also be initiated by peers. It serves to develop a "personal learning environment [A6]" that supports individual knowledge management based on a "needs assessment [A1]" and may be used for formal and informal learning formats. All success factors have to be implemented or proactively designed one after the other from [A1] through [A6] to maximize user centricity and create a personal learning environment that satisfies learning needs in the most effective way. The factors to be implemented sequentially are numbered in the design pattern [A] and marked with an arrow. Minimum requirements (MR) are shown in red (see Fig. 1).

\section{Design pattern $[\mathrm{B}]$ - Didactical variety}

Smart learning environments have to be didactically diverse (see section "Current state of research into smart learning environments") to enable effective teaching and learning. Design pattern (B) is the second phase of the design process; as such, it builds on the first phase's user centricity. The aim of the design pattern $[B]$ is to initiate the most effective learning processes within an SLE by combining different didactical models to

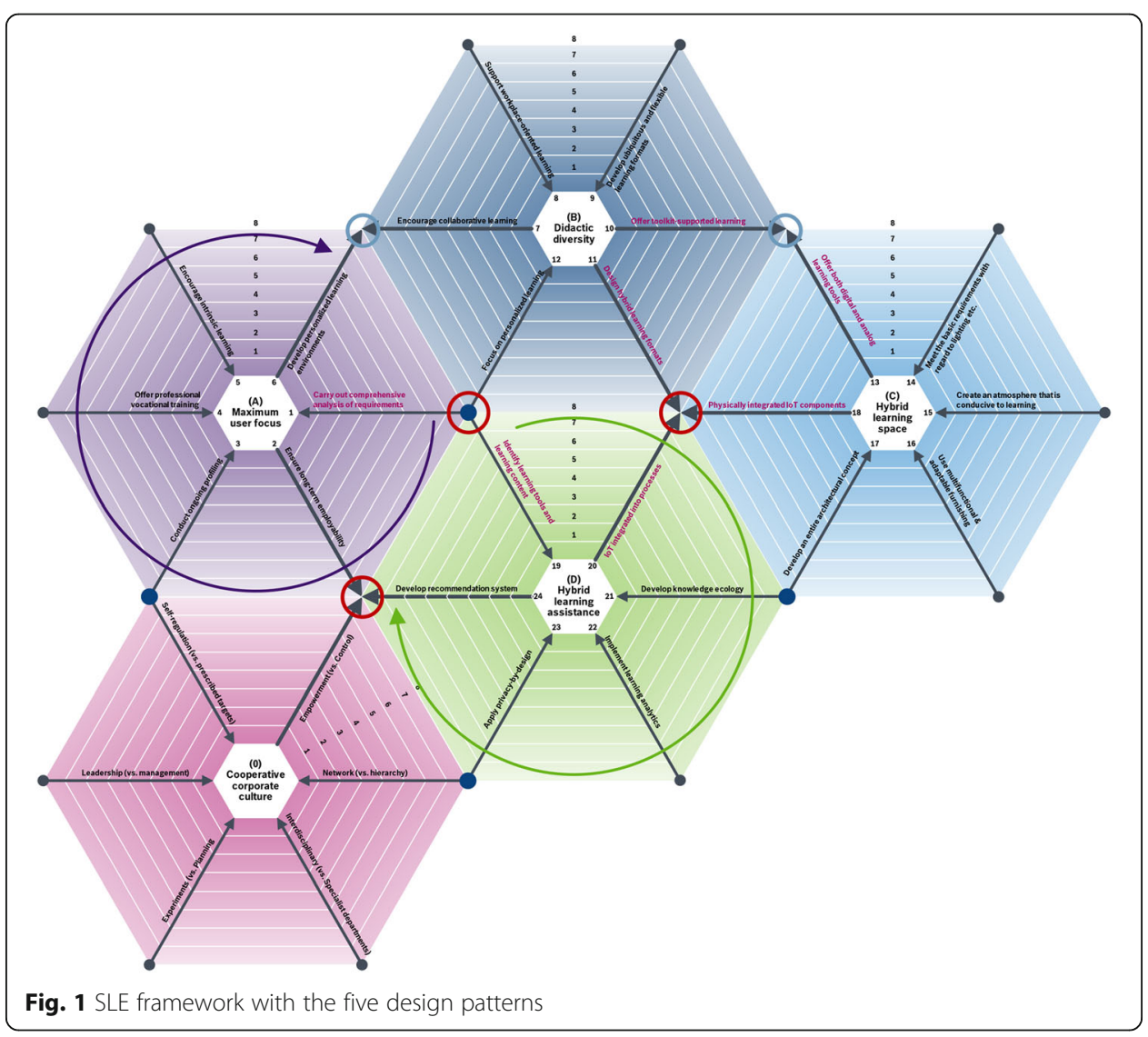


create a variety of methods. This study has shown that in the wake of changes in the continuing education sector, "workplace-oriented learning (B7)" has to be supported in order to satisfy fast-emerging and ad hoc learning needs as current events dictate. This is why highly "personalized learning (B12)" has to be reinforced to meet the demands of increasingly agile workflows. "Ubiquitous and adaptive forms of learning (B9)" that cater to learners' personal needs as well as to the current situation at the workplace or in the learning space must be developed to this end.

From a constructivist perspective, learning can never be controlled directly and always requires a proactive effort on the part the subject. Personalized learning is important. The results of this study show that "collaborative forms of learning (B8)" are also important. They cater to emerging learning needs that require exchanges and networking with other experts; as yet, these needs cannot be met due to a lack standardized learning formats, for instance via WBT and the like. New media such as Twitter and LinkedIn are particularly well-suited for this purpose. "Analog" methods such as Design Thinking are also suitable for promoting creativity, exchange and innovation. With active learning techniques such as creating sketches, drawings and simple models, several learners can simultaneously explore the problem and solution space. A variety of tools and materials need to be available for developing and trying out new forms of learning; this is a success factor for designing didactical variety. The underlying didactical model of "toolkit-supported learning" is success factor B10 and "hybridized learning" is success factor B11; both are defined as minimum requirements in design pattern (B). Hybridized forms of learning are learning processes where digital and physical learning methods alternate seamlessly and, in the best-case scenario, smart objects serve as fully integrated learning objects (e.g. a smart pen).

\section{Design pattern [C] - Hybrid learning space}

SLEs are physical spaces that are able to combine digital and physical learning worlds into hybrid environments (see section "Theoretical conclusions on SLEs, IoT and physical learning nodes"). Design pattern (C) is the third phase of the design process; as such, it builds on the second phase's didactical variety. The design process for hybrid learning spaces has to make use of ubiquitous but invisible IoT infrastructure. Co-working spaces serve as good examples for the physical design. The ambience affects the learning culture. It makes a difference if the learner happens to be in a grey office or is immersed in the sensual design of a Google Office. These extremes are meant to exemplify the point, but the designer does have to strike the right balance. According to the study, the most important success factor is to create a "learning-friendly atmosphere (C15)," which can be fostered with plenty of daylight, fresh air and sufficient shade, heating and cooling. Also, IoT integration should enable automated, (individual) control over these "standard requirements (C14)." Plenty of good-sized windows that admit a lot of daylight into the room are beneficial. Noise needs to be suppressed, for example, with soundproof walls.

The room's shapes, structures and colors should be part of a cohesive, "overall architectural concept (C17)" that inspires people to proactively learn and work as soon as they enter the room. A pleasant, modern design is to be combined with "multifunctional and flexible furniture (C16)" that readily adapts to different learning and working scenarios. 
Innovative concepts such as up-cycling could also serve to conjure an atmosphere that promotes learning and calls people to action (e.g. by means of tables made of pallets). The room's architectural concept - that is, the color, structure and ambience - should also blend in well with the neighboring environment (C17).

"Physically integrated IoT (C18)" and "digital \& analog learning tools (C13)" have been defined as key success factors for design pattern $(\mathrm{C})$ and as minimum requirements for designing hybrid learning spaces. Everyday objects can be transformed into "smart objects" using "physically integrated IoT (C18)". This means enhancing everyday objects such as windows with sensors and actuators to create an added, automated benefit. The technology recedes inconspicuously into the background. Integrated into walls, tables, chairs and so forth, it becomes part of the architecture and furniture. Examples include in-built speakers in walls, sofas and the like and multifunctional room dividers that serve as a touch screen when needed and can otherwise be used as a shelf or writable wall. The second minimum requirement, the "digital and analog learning tools (C13)" described in the design pattern (B) for didactical variety, have to be accessible in the physical learning room to allow learners to do things like launching software programs from their PLE without having to first install it.

\section{Design pattern [D] - Learning assistance}

SLEs provide demand- and context-driven learning assistance (cf. section "Current state of research into smart learning environments"). Pattern (D) for designing a hybrid learning assistant is the SLE design process's overall objective. Building on previous design patterns, it is the final and most complicated phase. Design pattern (D)'s success factors must be implemented sequentially to ensure the (hybrid) learning assistant provides the functions needed to deliver optimum learning assistance via a "recommender system (D24)." The factors to be implemented sequentially are numbered in the design pattern $[\mathrm{D}]$ and marked with an arrow.

This sequence of success factors begins with factor (D19), whereby learning objects and learning content based on factor A1 are to be explicated and documented even more specifically. Designers must first systematically "identify current (and future) learning objects and learning content (D19)" before they can move on to the second step and integrate the Internet of Things technology construct into the identified learning objects, in order for them to effectively assist the learning process. Specific learning content and learning processes must also be defined to then build on the physically integrated IoT (C18) to create a "process-based integrated IoT (D20)." The third success factor is to foster a "knowledge ecology (d21)." Serving as a knowledge database for the assistant, it may be comprised of various resources and databases. However, the relevant learning resources and knowledge sources must first be identified, made interoperable and be interconnected. It would be a decisive advantage for PLE data (A6) to be used to this end. The fourth step is to apply "big data analytics (d22) \& privacy by design (D23)" to establish sound connections between the various knowledge bases and data sources in compliance with data privacy obligations and laws. Artificial intelligence-driven processes such as machine learning must serve to support learning activities that have not been programmed in advance. The learning assistant is thus able to identify the current learning situation based on usage data and recognized context. 
It can then decide what option works best for learners based on predictive analytics (D22) that assess past situations, and to respond proactively in the spirit of a "recommender system (D24)".

\section{Limitations}

This study is based on various data surveys conducted at different points in time (sub study 1 , sub study 2 and main study). Given the scope of this paper and in the interest of brevity, they have been merely outlined (cf. section "Current state of research into smart learning environments"). Nonetheless, the most important limitations are pointed out below. This scientific treatise is subject to certain limitations, so that its results cannot be held to be universally valid in every context. In particular, it should be noted that sub study 1 with IoT trainers and the main study, which included sub study 2 , were conducted predominantly with university graduates in senior management positions serving as the research subjects. Theirs is a very specific perspective. A survey of teachers and academics from less knowledge-intensive areas of business or science may have resulted in to different findings. The results obtained using the quantitative survey tool may only be interpreted as an initial tendency because the very small sample size limits their informative value.

\section{Discussion}

The findings of this research paint a comprehensive picture of IoT-based innovations in learning that have otherwise been researched in separate specialized disciplines and posited in compartmentalized concepts. The contribution of the proposed framework results within a transdisciplinary combination of different SLE design approaches in one overall concept. Thus, substantial challenges in previous SLE research could be addressed by systematically breaking up the unilaterally developed concepts and by holistically considering involved disciplines, design areas and success factors. Architectural design principles as well as organizational aspects were purposefully analyzed and interwoven with didactic and information technology SLE characteristics.

The study provides an important contribution to the theoretical recognition of SLE research. Thus, the SLE model was validated and re-de-signed based on the empirical data. This study is the first of its kind that can address the application of SLEs for workplace learning and can be used as an analysis and planning tool. In addition, the framework provides concrete SLE design recommendations for further training experts, which are bundled within 5 categories and 30 success factors and which reveal clear cross-references to one another. The identification of the success factors and the assignment to a coherent category system has been validated empirically and can serve as a basis for future work in the field of Smart Learning Environments research. It should be noted that the re-design of the design patterns as well as the merge into an SLE framework were not re-validated. A stronger differentiation and operationalization of the individual categories and factors implies new research potential. The framework based on probabilistic relationships offers correspondingly great potential for constructive research that investigates causal relationships on the basis of factor analysis using multivariate statistics. 


\section{Conclusion and future research}

There is great demand for further (empirical) and interdisciplinary research into smart learning environments. An extensive review of the literature has shown that IoT-based educational formats are a neglected field of research. Sound research into the application of teaching and learning processes is not to be found in the scientific discourse at home in Germany or abroad. A lack of empirical findings also points to the fact that studies have yet to be conducted, even if the Internet of Things has been a hot topic in recent years.

This research project sought to conceptualize a largely uniform ontological basis with defined categories to serve as recommendations for action within the framework of organizational SLE design processes. Its authors redressed the insufficient systematization of earlier SLE research by developing design patterns for the constructs "user centricity (A)", "didactical variety (B)", "hybrid learning space $(\mathrm{C})$ " and "hybrid learning assistance (D)." The identified success factors and their allocation to a cohesive system of categories has been empirically validated and can serve as a starting point for future research into smart learning environments. It should be noted that the interaction between technologies and (new) teaching and learning formats must always focus on creating added didactical value. The applied technology is a means to an end and no substitute for the underlying objective. Learning is a complex process in which emotions and intrinsic motivations are decisive factors that defy direct control. This paper combines framework conditions that are indirectly conducive to the didactically sound design of SLEs. Its authors identified 30 factors that can be addressed to support a systematic design process for innovative forms of education. This is an ideal design process. Specifications set out by this paper may be difficult to meet in the real world of business.

However, the focus must always be on the users' needs, especially in terms of didactical variety. Learning has to made more varied and active to appeal to the emotions and foster creativity so that it is perceived as a joyful rather than a tedious experience.

These findings support the thesis that an interdisciplinary approach is expedient when designing smart learning environments. Interdisciplinary insights have to be pooled to understand hybrid constructs as complex as this and to design sound SLEs. Future research into SLEs will have to consider and bring neighboring disciplines on board to investigate causal relationships at the meta-level. These findings also point to critical aspects. For example, personal data must be handled in a very cautious and user-centric way.

Funding

The authors declare that they have no funding for the research.

Availability of data and materials

The datasets used and/or analysed during the current study are available from the corresponding author on reasonable request.

Authors' contributions

The main author (SF) performed all research and writing of the paper. LS and TK provided insight and editing of the paper. All authors read and approved the final manuscript. 


\section{Publisher's Note}

Springer Nature remains neutral with regard to jurisdictional claims in published maps and institutional affiliations.

\section{Author details}

${ }^{1}$ Bosch Software Innovations, Berlin, Germany. ${ }^{2}$ Dresden University of Technology, Institute for Vocational Education, Dresden, Germany.

Received: 13 June 2018 Accepted: 12 October 2018

Published online: 24 October 2018

\section{References}

E. Aarts, R.S. Harwig, in The invisible future: The seamless integration of technology in everyday life. Ambient intelligence (2002), pp. 235-250

M. Ashcraft, G. Radvansky, Cognition (Pearson Education, UpperSaddleRiver, 2010)

P. Brusilovsky, C. Peylo, Adaptive and intelligent web-based educational systems. Int. J. Artif. Intell. Educ (IJAIED). 13, 159-172 (2003)

M. G. Domingo, Forner, J. A. M. Expanding the Learning Environment: Combining Physicality and Virtuality - The Internet of Things for eLearning (S. 730-731). IEEE. (2010) https://doi.org/10.1109/ICALT.2010.211

S. Doorley, S. Witthoft, Make Space: How to Set the Stage for Creative Collaboration (John Wiley \& Sons, Hoboken, N.J, 2012)

M. Ebner, S. Schön, BIMS, Lehrbuch für Lernen und Lehren mit Technologien: [L3T] ([Stand:] Mai 2011) (Books on Demand, Norderstedt, 2011)

O. Eigenbrod, R. Stang, Formierungen von Wissensräumen: Optionen des Zugangs zu Information und Bildung (De Gruyter Saur, Berlin; Boston, 2014)

J. Erpenbeck, W. Sauter, So werden wir lernen! Kompetenzentwicklung in einer Welt fühlender Computer, kluger Wolken und sinnsuchender Netze (Springer Berlin Heidelberg, Berlin, Heidelberg, 2013)

J. Erpenbeck, W. Sauter, Kompetenzentwicklung mit humanoiden Computern. Die Revolution des Lernens via Cloud Computing und semantischen Netzen (Springer Fachmedien Wiesbaden, Wiesbaden, 2015)

D. Estrin, D. Culler, K. Pister, G. Sukhatme, Connecting the physical world with pervasive networks. IEEE Pervasive Computing 1(1), 59-69 (2002)

E. Fleisch, Thiesse, F. Internet der Dinge - Enzyklopädie der Wirtschaftsinformatik [Lehrstuhl für Wirtschaftsinformatik und Electronic Government, Universität Potsdam] (2014). http://www.enzyklopaedie-der-wirtschaftsinformatik.de/wienzyklopaedie/lexikon/technologien-methoden/Rechnernetz/Internet/Inter-net-der-Dinge/index.html/?searchterm= internet\%20der\%20dinge. Accessed 20 Aug 2015

IML. Fraunhofer. Was ist das Internet der Dinge? (2018) https://www.internet-der-dinge.de/. Accessed 18 Oct 2018

P. Gabriel, K. Gaßner, \& S. Lange, Das Internet der Dinge - Basis für die IKT-Infrastruktur von morgen Anwendungen, Akteure und politische Handlungsfelder. Institut für Innovation und Technik (iit) in der VDINDE-IT (2010).

J. Gómez, J.F. Huete, O. Hoyos, L. Perez, \& D. Grigori, Interaction System based on Internet of Things as Support for Education. Procedia Computer Science, 21, 132-139 (2013). Accessed 19 Nov. 2016, https://doi.org/10.1016/j.procs.2013.09.019.

B. Gros, The design of smart educational environments. Smart Learning Environments 3(1) (2016). https://doi.org/10.1186/ s40561-016-0039-x

A.R. Hevner, S.T. March, J. Park, S. Ram, Design science in information systems research. MIS Q. 28(1), 75-105 (2004)

G.-J. Hwang, Definition, framework and research issues of smart learning environments - a context-aware ubiquitous learning perspective. Smart Learning Environments 1(1) (2014). https://doi.org/10.1186/s40561-014-0004-5

S. Kanagarajan, S. Ramakrishnan, Ubiquitous and ambient intelligence assisted learning environment infrastructures development - a review. Educ. Inf. Technol. (2017) https://doi.org/10.1007/s10639-017-9618-x

M. Kerres. E-Learning vs. Digitalisierung der Bildung: Neues Label oder neues Paradigma? In: A. Hohenstein, \& K. Wilbers (Hrsg.) Handbuch E-Learning, Köln: Fachverlag Deutscher Wirtschaftsdienst, Ergänzungslieferung, ISBN 978-3-87156-298-3, 61 (2016)

J.H. Knoll, Architektur und Erwachsenenbildung, in Internationales Jahrbuch der Erwachsenenbildung 23, (Böhlau, Köln, 1995)

C. Kohls, G. Münster, in Bildungsräume 2017 (S. 39-50), ed. by C. Igel, C. Ullrich, W. Martin. Hybride Lernräume für Innovationsprozesse (Gesellschaft für Informatik, Bonn, 2017)

R. Koper, Conditions for effective smart learning environments. Smart Learning Environments 1(1), 1-17 (2014)

C.-U. Lei, K. Wan, K.L. Man, Developing a smart learning environment in universities via cyber-physical systems. Procedia Computer Science 17, 583-585 (2013) https://doi.org/10.1016/j.procs.2013.05.075

U. Lucke, C. Rensing, A survey on pervasive education. Pervasive and Mobile Computing 14, 3-16 (2014)

F. Mattern, E. Fleisch, Das Internet der Dinge. Ubiquitous Computing und RFID in der Praxis: Visionen, Technologien, Anwendungen, Handlungsanleitungen. (Springer-Verlag, Berlin Heidelberg, 2005)

P. Mayring, Qualitative Inhaltsanalyse. Handbuch qualitative Forschung in der Psychologie, 601-613Sesink, W. (2007). Raum und Lernen. Education Permanente. Schweizerische Zeitschrift für Weiterbildung 41(1), 16-18 (2010)

J.M. Spector, Conceptualizing the emerging field of smart learning environments. Smart Learning Environments 1(1), 1-10 (2014)

W. Sesink, Raum und Lernen. Education Permanente. Schweizerische Zeitschrift für Weiterbildung, 41(1), 16-18 (2007).

H. Stachowiak, Allgemeine Modelltheorie. (Springer-Verlag, Wien, New York, 1973)

A. Uzelac, N. Gligoric, S. Krco, A comprehensive study of parameters in physical environment that impact students' focus during lecture using internet of things. Comput. Hum. Behav. 53, 427-434 (2015)

O. Vermesan, Friess, P. Building the Hyperconnected Society - Internet of Things Research and Innovation Value Chains, Ecosystems and Markets. (River Publishers, Aalborg, 2015)

M. Weiser, The computer for the 21 st century. Sci. Am. 265(3), 94-105 (1991)

T. Winkler, F. Scharf, M. Herczeg, Ambiente Lernräume: Lernen mit vernetzten, interaktiven, körper- und raumbezogenen Medien. Informatik-Spektrum 37(5), 445-448 (2014) https://doi.org/10.1007/s00287-014-0817-1

Z.-T. Zhu, M.-H. Yu, P. Riezebos, A research framework of smart education. Smart Learning Environments 3(1) (2016). https:// doi.org/10.1186/s40561-016-0026-2 